\title{
Biotechnology 2000
}

\author{
Aris Persidis
}

With biotech beginning to show signs of a recovery in the latter half of 1999, the year's end is a good opportunity to extend our positive thinking and contemplate which areas and technologies might grow considerably in the coming year. This has been a tradition since this column's inception more than two years ago. It continues here, focusing on five technologies and areas that show some important signs of growth for the future.

The choice is, of course, vast, and there are many technologies and applications that deserve to be highlighted. The technologies and applications discussed here were chosen on the basis of major recent advances that have invented or re-invented them and opened up very significant markets as a result.

\section{Technologies to watch}

Computer simulation-based biology. There is such a vast amount of biological data available today, including annotated databases of pathophysiology, pharmacogenomics, quantitative structure-activity relationships (QSAR), genomic and proteomic information, and cell, tissue and organ-specific responses, that it stands to reason that correlational and statistical analysis of all this information could be performed with the goal of generating predictive models and biological systems that reside entirely within computers, which would have real value in all phases of the discovery and development of new drugs. This is indeed being pursued by a few companies, and right now there are computer models and simulations of drug metabolism, asthma, obesity, HIV/AIDS, pharmacokinetics, human organs such as the heart and the human gastrointestinal tract, and also even clinical trial modeling.

Pro: very low cost to develop and implement. Con: requires extensive real-world validation.

Bioinformatics. In addition to databasedriven simulations, bioinformatics is widely acknowledged to be a core capability whose technology base, applications, and impact is far from being realized. Already, significant value has been generated for early movers here, and novel algorithms, applications, and business models will be seen in the near future. Fueling this is the tidal wave of "bioinformation" emanating from genomics,

Aris Persidis is managing director of RHeoGene, 706 Forest Street, Charlottesville, VA 22903 (apersidis@rheogene.com). proteomics, and variants of these approaches. Sequence analysis of every form and correlations with all existing annotations in the public and private databases is being orchestrated around the industry, and this will facilitate the identification and validation of novel drug targets in humans as well as in plants and animals.

Pro: cheap to implement.

Con: many players, non-standardized approaches.

Tissue engineering. Tissue and organ failure, produced as a result of injury or other type of damage, is a major health problem of multi-billion dollar proportions, accounting for about half of the total annual expenditure in health care in the US. The options for treatment are surgery, artificial limbs, organ transplantation (human or xenotransplantation), mechanical devices, and in a few cases, drug therapy. Ultimately, however, major damage to a tissue or organ cannot be repaired. The emergence of tissue engineering as a potential alternative or complementary solution is a watershed for these conditions. Although initial efforts have focused on skin equivalents for treating burns, there is a significant pipeline being developed of engineered tissues, and biomaterials and scaffolds used to deliver them. Notable results include tissue-engineered bone, blood vessels, liver, muscle, and nerve conduits.

Pro: vast market, significant space for new technologies.

Con: difficult and expensive.

Gene switching. The ability to preceisely control gene expression via a "switch", i.e., a ligand-receptor complex that permits transcription to occur, has been around for about a dozen years, with well-known small molecules, such as tetracycline, rapamycin, and ecdysone analogs being used this way. The primary application of gene switching is in gene therapy, specifically in cases where modulation of gene expression is desired, as opposed to a permanent "gene on" situation. In addition, switching has the added benefit of providing a safety mechanism in gene therapy. Recent advances also show how gene switching can be used in functional genomics and screening, opening up significant areas of application and markets for these technologies.

Pro: Untapped potential in multiple applications.

Con: Targeting and validation difficulties.

Molecular cardiovasular disease drug discovery. Cardiovascular diseases cause 15 mil- lion deaths annually worldwide, and a disease burden in the US alone of $\$ 150$ billion. Although there are many procedures and medicines used today, cardiovascular medicine is constantly evolving and taking advantage of new opportunities. In addition to improved surgical procedures and drugs, cardiovascular medicine is beginning to use genomic information, ushering in the field of molecular cardiovascular medicine. For example, expressed sequence tags (ESTs) from cDNA libraries of the cardiovascular system are being used to identify widely expressed and tissue, development-stage or disease-stage specific genes. This capability will no doubt result in improved understanding of molecular contributors, which in turn will help design better new drugs.

Pro: huge market.

Con: long-term horizon required.

\section{Conclusions}

As the millennium finally closes and biotechnology enters its fourth decade, it is important to note that all the technologies and applications described here and throughout this column the past few years show conclusively that the field is constantly re-inventing itself. This is the direct result of new advances in assays, reagents, and instrumentation, which in combination lead to deeper and better knowledge of basic as well as disease mechanisms.

In addition, the computer is playing an ever-important role, especially as there are enough databases and information now to begin to discern meaningful trends in biological responses and mechanisms. These approaches will translate themselves into new therapeutics and diagnostics in the near future, at a cost far less than was previously the norm, specifically for the portion of preclinical development that revolves around extensive in vitro and animal studies. Hence, the continued ascent of bioinformatics and the arrival of simulation biology are noted here.

In addition, other technologies such as tissue engineering, gene switching, and molecular medicine of the heart are receiving huge benefits from recent technical advances, which have no less than reinvented these areas as essentially new ones. Investors are already returning to biotech, because of these and many other advances, and this is the best entrance into the new millennium that we as practitioners could have hoped. 